\title{
Isobutyraldehyde production from Escherichia coli by removing aldehyde reductase activity
}

\author{
Gabriel M Rodriguez and Shota Atsumi*
}

\begin{abstract}
Background: Increasing global demand and reliance on petroleum-derived chemicals will necessitate alternative sources for chemical feedstocks. Currently, 99\% of chemical feedstocks are derived from petroleum and natural gas. Renewable methods for producing important chemical feedstocks largely remain unaddressed. Synthetic biology enables the renewable production of various chemicals from microorganisms by constructing unique metabolic pathways. Here, we engineer Escherichia coli for the production of isobutyraldehyde, which can be readily converted to various hydrocarbons currently derived from petroleum such as isobutyric acid, acetal, oxime and imine using existing chemical catalysis. Isobutyraldehyde can be readily stripped from cultures during production, which reduces toxic effects of isobutyraldehyde.

Results: We adopted the isobutanol pathway previously constructed in E. coli, neglecting the last step in the pathway where isobutyraldehyde is converted to isobutanol. However, this strain still overwhelmingly produced isobutanol (1.5 g/L/OD 600 (isobutanol) vs $0.14 \mathrm{~g} / \mathrm{L} / \mathrm{OD}_{600}$ (isobutyraldehyde)). Next, we deleted yqhD which encodes a broad-substrate range aldehyde reductase known to be active toward isobutyraldehyde. This strain produced isobutanol and isobutyraldehyde at a near 1:1 ratio, indicating further native isobutyraldehyde reductase (IBR) activity in E. coli. To further eliminate isobutanol formation, we set out to identify and remove the remaining IBRs from the E. coli genome. We identified 7 annotated genes coding for IBRs that could be active toward isobutyraldehyde: adhP, eutG, yiaY, yjgB, betA, fucO, eutE. Individual deletions of the genes yielded only marginal improvements. Therefore, we sequentially deleted all seven of the genes and assessed production. The combined deletions greatly increased isobutyraldehyde production $\left(1.5 \mathrm{~g} / \mathrm{L} / \mathrm{OD}_{600}\right)$ and decreased isobutanol production $\left(0.4 \mathrm{~g} / \mathrm{L} / \mathrm{OD}_{600}\right)$. By assessing production by overexpression of each candidate IBR, we reveal that AdhP, EutG, YjgB, and FucO are active toward isobutyraldehyde. Finally, we assessed long-term isobutyraldehyde production of our best strain containing a total of 15 gene deletions using a gas stripping system with in situ product removal, resulting in a final titer of $35 \mathrm{~g} / \mathrm{L}$ after 5 days.

Conclusions: In this work, we optimized E. coli for the production of the important chemical feedstock isobutyraldehyde by the removal of IBRs. Long-term production yielded industrially relevant titers of isobutyraldehyde with in situ product removal. The mutational load imparted on E. coli in this work demonstrates the versatility of metabolic engineering for strain improvements.
\end{abstract}

\section{Background}

The dependence on finite petroleum and natural gas resources as well as their potential environmental impact has generated interest in exploring renewable sources for replacements. This has more notably been applied to the areas of transportation fuels. However, less attention has been paid to the chemical feedstock industry.

\footnotetext{
* Correspondence: satsumi@ucdavis.edu

Department of Chemistry, University of California, One Shields Ave, Davis, CA 95616, USA
}

(c) 2012 Rodriguez and Atsumi; licensee BioMed Central Ltd. This is an Open Access article distributed under the terms of the Creative Commons Attribution License (http://creativecommons.org/licenses/by/2.0), which permits unrestricted use, distribution, and reproduction in any medium, provided the original work is properly cited. chemical industry consumed 4 quadrillion BTUs (British thermal units) of petroleum and natural gas for feedstock use to produce thousands of chemicals [1]. These chemicals are essential to the synthesis of plastics, rubbers, and pharmaceutical compounds that play a major role in our standard of living.

Synthetic biology has made large progress constructing pathways for the production of various biofuels [2-5]. Recently, these efforts have expanded to address the 
need for replacement of our current petrochemical feedstocks with renewable sources [6-13]. Much like in the production of advanced biofuels, synthetic biology offers a potential platform for the production of nonnatural chemical feedstocks from simple sugars. This work aims to demonstrate the feasibility of producing chemical feedstocks from microorganisms by engineering Escherichia coli to produce isobutyraldehyde.

Isobutyraldehyde is used as both a fragrance and flavor additive. It is also used to produce plasticizers, isobutyric acid, and isobutanol, which is a precursor to the rubber polyisobutylene. Isobutyraldehyde is currently synthesized from petroleum derived propylene, carbon monoxide, and hydrogen [14]. In terms of microbial production and purification, its high volatility $\left(172 \mathrm{~mm} \mathrm{Hg}\right.$ at $\left.25^{\circ} \mathrm{C}\right)$ may enable less costly purification and facilitate in situ product removal for long-term fermentation.

Atsumi et al. have previously shown that 2ketoisovalerate generated from L-valine biosynthesis can serve as precursors for the Ehrlich degradation pathway [15] to isobutanol (Figure 1) [5]. In this pathway, 2ketoisovalerate is converted to isobutyraldehyde using a keto acid decarboxylase (KDC) and then reduced to isobutanol with an isobutyraldehyde reductase (IBR). In 2010, Atsumi et al. evaluated the native aldehyde reductase YqhD for its ability to convert isobutyraldehyde to isobutanol in $E$. coli [16]. It was discovered that a strain lacking overexpression of IBR was still able to produce the same amount of isobutanol as a strain overexpressing ADH2 (Saccharomyces cerevisiae). It was determined that YqhD in E. coli was responsible for most of IBR activity in isobutanol production. However, even with deletion of $y q h D$ on the E. coli chromosome, the engineered strain was still able to produce isobutanol. It indicates that $E$. coli has one or several additional IBRs. Thus, here, we systematically removed possible IBRs from the $E$. coli chromosome to increase isobutyraldehyde production and reduce isobutanol formation.

\section{Results and discussion}

As a starting point, E. coli strain JCL260 was used to assess initial isobutyraldehyde production. This strain was previously optimized for isobutanol production [5,17], by deleting adhE, ldhA, fnr, frdAB, pta, and $p f l B$. The isobutyraldehyde pathway was constructed by using the previously described genes alsS, ilvC, ilvD, and kivd [5,18] .

To assess the initial production, pGR03 (alsS, ilvC, and $i l v D$ ) and pSA129 (kivd) were introduced into JCL260. This strain produced only $0.14 \mathrm{~g} / \mathrm{L} / \mathrm{OD}_{600}$ isobutyraldehyde, and as high as $1.45 \mathrm{~g} / \mathrm{L} / \mathrm{OD}_{600}$ isobutanol after 24 hours. This roughly 1:10 ratio is likely the result of one or several IBRs including $y q h D$ on the E. coli genome [16]. As a result, we first deleted $y q h D$. This strain produced $\sim 0.7 \mathrm{~g} / \mathrm{L} / \mathrm{OD}_{600}$ of isobutyraldehyde and $\sim 0.4 \mathrm{~g} / \mathrm{L} / \mathrm{OD}_{600}$

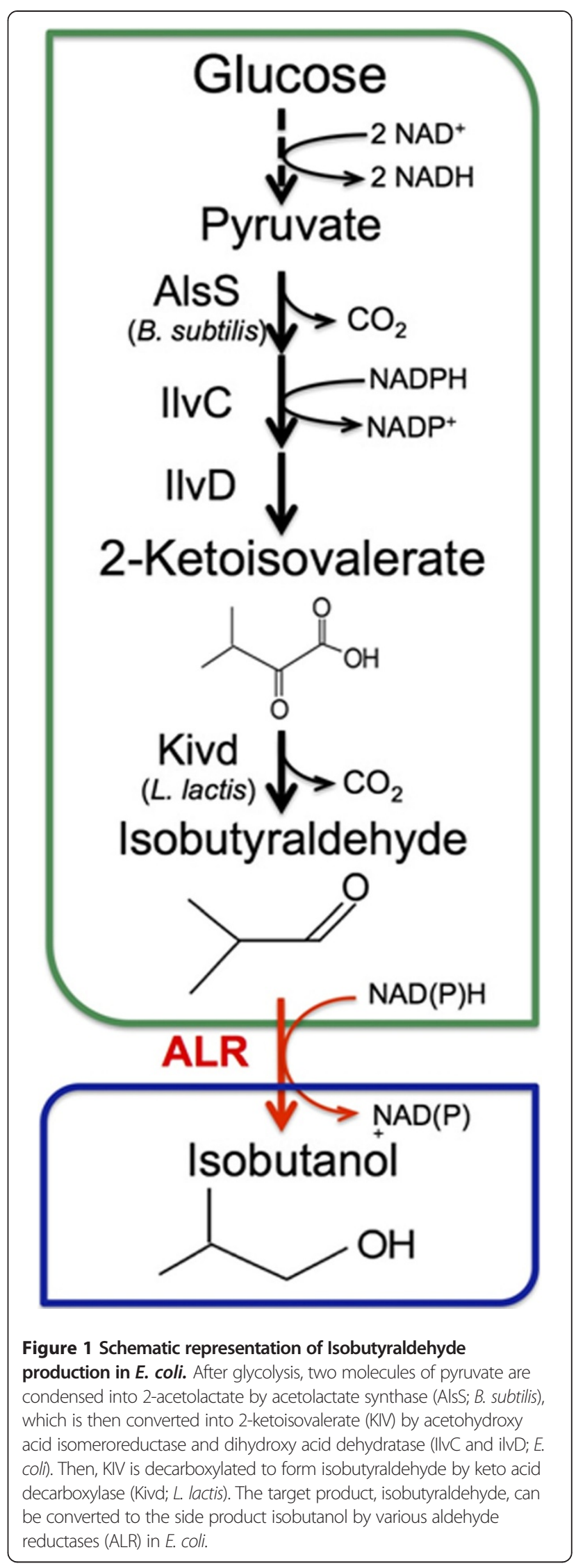


isobutanol in our condition. This result indicates one or more additional $I B R$ s exist on the $E$. coli genome.

IBR activity can be catalyzed by the alcohol dehydrogenase $(\mathrm{ADH}) /$ aldehyde reductase class of enzyme. Thus, in order to decrease isobutanol production, we searched the E. coli genome sequence using the EcoCyc comprehensive database [19] for other ADH-like enzymes. We identified seven candidate IBRs (adhP, eutG, yiaY, yjgB, betA, fucO, and eutE). Due to either the characterized ADH functions or the conserved $\mathrm{ADH}$-like domain sequences of the above-mentioned genes, we hypothesized that these had the highest probability of having IBR activity. Additionally, no other ADH type enzymes were found. AdhP is a wellcharacterized NADH-dependent ethanol dehydrogenase [20]. EutG is an NADH-dependent alcohol dehydrogenase involved in ethanolamine utilization, and is suspected to be an ethanol dehydrogenase [21-23]. The enzymes EutG, YiaY, BetA, and EutE were previously utilized or removed to produce C4-C10 alcohols [24]. FucO has also been well characterized as a L-lactaldehyde and 1,2-propanediol oxidoreductase [25]. YjgB is a putative Zn-dependent aldehyde reductase [26]. With seven candidate genes identified, we set out to efficiently identify which (if any) of these enzymes were active toward isobutyraldehyde in order to improve isobutyraldehyde production and reduce isobutanol formation.

\section{Single gene deletions to improve isobutyraldehyde production}

As a first step, each candidate $I B R$ gene was individually deleted on the $\Delta y q h D$ strain (SA542 (Table 1)). Then, we measured production by introducing pGR03 (alsS, ilvC, and $i l v D$ ) and pSA129 (kivd) into each strain (Figure 2A). Production was carried out in sealed screw-cap culture tubes to prevent evaporation of isobutyraldehyde. None of the individual genes stood out as largely responsible for the observed isobutanol production (Figure 2A). However, two strains ( $\triangle y i a Y$ and $\triangle y j g B$ ) showed slight improvements in isobutyraldehyde (up to $1 \mathrm{~g} / \mathrm{L} / \mathrm{OD}_{600}$ ) over the $\Delta y q h D$ strain $\left(0.7 \mathrm{~g} / \mathrm{L} / \mathrm{OD}_{600}\right)$, while two $(\Delta b e t A$ and $\Delta e u t E$ ) showed decreases to below $0.4 \mathrm{~g} / \mathrm{L} / \mathrm{OD}_{600}$. We considered that multiple enzymes could be involved in IBR activity, resulting in minor or no observable changes in production. In order to test this hypothesis, we set out to combine the deletions and assess production with each additional deletion. If several enzymes were responsible, then total elimination of these genes would result in higher isobutyraldehyde production, and little or no isobutanol formation.

\section{Combining gene deletions to improve isobutyraldehyde production}

Next we sequentially combined all candidate $I B R$ deletions. With each additional deletion we measured production of
Table 1 Strain and plasmids used in this work

\begin{tabular}{|c|c|c|}
\hline Name & Genotype & Reference \\
\hline BW25113 & $\begin{array}{l}r r n B_{\mathrm{T14}} \Delta \operatorname{lac}_{\mathrm{a} Z \mathrm{WJ} 16} \text { hsdR514 } \\
\Delta \text { araBAD }_{\mathrm{AH} 33} \Delta r h a B A D_{\mathrm{LD} 78}\end{array}$ & [27] \\
\hline JCL16 & BW25113/F' [traD36, proAB ${ }^{+}$, lacl $\left.{ }^{q} Z \Delta M 15\right]$ & [17] \\
\hline JCL260 & JCL16: $\Delta a d h E ; \Delta f r d B C ; \Delta p t a ; \Delta f n r-l d h A ; \Delta p f l B$ & {$[5]$} \\
\hline SA542 & JCL260: $\Delta y g h D$ & [16] \\
\hline AL287 & SA542: $\triangle a d h P$ & This work \\
\hline AL288 & SA542: $\Delta$ eutG & This work \\
\hline AL289 & SA542: $\Delta$ yiaY & This work \\
\hline AL290 & SA542: $\Delta y j g B$ & This work \\
\hline AL555 & SA542: $\Delta$ betA & This work \\
\hline AL615 & SA542: $\Delta$ fucO & This work \\
\hline AL616 & SA542: $\triangle$ eutE & This work \\
\hline AL312 & SA542: $\triangle$ adhPDeutG & This work \\
\hline AL322 & SA542: $\triangle a d h P \Delta e u t G \Delta y i a Y$ & This work \\
\hline AL329 & SA542: $\Delta a d h P \Delta e u t G \Delta y i a Y \Delta y j g B$ & This work \\
\hline AL556 & SA542: $\Delta a d h P \Delta e u t G \Delta y i a Y \Delta y j g B \Delta b e t A$ & This work \\
\hline AL626 & 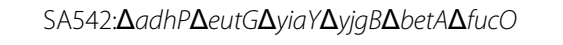 & This work \\
\hline AL707 & 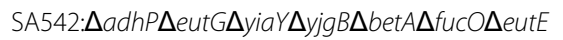 & This work \\
\hline
\end{tabular}

\begin{tabular}{|c|c|c|}
\hline Plasmids & Features & Reference \\
\hline pSA69 & p15A ori; Kan ${ }^{R} ; P_{L} l a c O_{1}$ : alsS-ilvCD & [5] \\
\hline pSA129 & ColE1 ori; $\mathrm{Amp}^{\mathrm{R}} ; P_{\mathrm{L}} \mathrm{lacO}_{1}$ : kivd & [16] \\
\hline pSA138 & ColE1 ori; $P_{\mathrm{L}}$ lacO 1 : kivd-yqhD & {$[5]$} \\
\hline pGR03 & p15A ori; $\mathrm{Cm}^{\mathrm{R}} ; P_{\mathrm{L}} \mathrm{lacO}_{1}$ : alss-ilvCD & This work \\
\hline pAL217 & ColE1 ori; $\mathrm{Amp}^{\mathrm{R}} ; P_{\mathrm{L}} \mathrm{lacO}_{1}$ : kivd-adhP & This work \\
\hline pAL218 & ColE1 ori; Amp $; P_{L}$ lacO $_{1}$ : kivd-eutG & This work \\
\hline pAL219 & ColE1 ori; $\mathrm{Amp}^{\mathrm{R}} ; \mathrm{P}_{\llcorner} \mathrm{lacO}_{1}$ : kivd-yiaY & This work \\
\hline pAL220 & ColE1 ori; $\mathrm{Amp}^{\mathrm{R}} ; P_{\mathrm{L}} \mathrm{lacO}_{1}$ : kivd-yjgB & This work \\
\hline pAL221 & ColE1 ori; $\mathrm{Amp}^{\mathrm{R}} ; P_{\mathrm{L}} \mathrm{lacO}_{1}$ : kivd-betA & This work \\
\hline pAL222 & ColE1 ori; $\mathrm{Amp}^{\mathrm{R}} ; P_{\mathrm{L}} \mathrm{lacO}_{1}$ : kivd-fucO & This work \\
\hline pAL223 & ColE1 ori; $\mathrm{Amp}^{\mathrm{R}} ; P_{\mathrm{L}} \mathrm{lacO}_{1}$ : kivd-eutE & This work \\
\hline pZE12-luc & ColE1 ori; $\mathrm{Amp}^{\mathrm{R}} ; P_{\mathrm{L}}$ lacO 1 : luc(VF) & [28] \\
\hline pAL162 & ColE1 ori; $\mathrm{Amp}^{\mathrm{R}} ; P_{\mathrm{L}} \mathrm{lacO}_{1}: \operatorname{adhP}$ & This work \\
\hline pAL158 & ColE1 ori; $\mathrm{Amp}^{\mathrm{R}}$; $P_{\mathrm{L}}$ lacO 1 : eutG & This work \\
\hline pAL157 & ColE1 ori; $\mathrm{Amp}^{\mathrm{R}} ; P_{\mathrm{L}} \mathrm{lacO}_{1}$ : yiaY & This work \\
\hline pAL156 & ColE1 ori; $\mathrm{Amp}^{\mathrm{R}} ; P_{\mathrm{L}} \mathrm{lacO}_{1}:$ yjgB & This work \\
\hline pAL213 & ColE1 ori; $\mathrm{Amp}^{\mathrm{R}} ; P_{\mathrm{L}} \mathrm{lacO}_{1}$ : betA & This work \\
\hline pAL214 & ColE1 ori; $\mathrm{Amp}^{\mathrm{R}} ; P_{\mathrm{L}} \mathrm{lacO}_{1}$ : fucO & This work \\
\hline pAL215 & ColE1 ori; $\mathrm{Amp}^{\mathrm{R}} ; P_{\mathrm{L}} \mathrm{LacO}_{1}$ : eutE & This work \\
\hline
\end{tabular}

isobutyraldehyde and isobutanol (Figure 2B). The isobutyraldehyde production from the strain including $\triangle a d h P \Delta e u t G$ and $\Delta y i a Y$ was similar with that from the parent strain $(\Delta y q h D)$. However, with $\triangle y j g B$ added to the strain including $\Delta y q h D \triangle a d h P \Delta e u t G$ and $\Delta y i a Y$ we observe a significant drop in isobutanol production to $0.19 \mathrm{~g} / \mathrm{L} / \mathrm{OD}_{600}$ and 

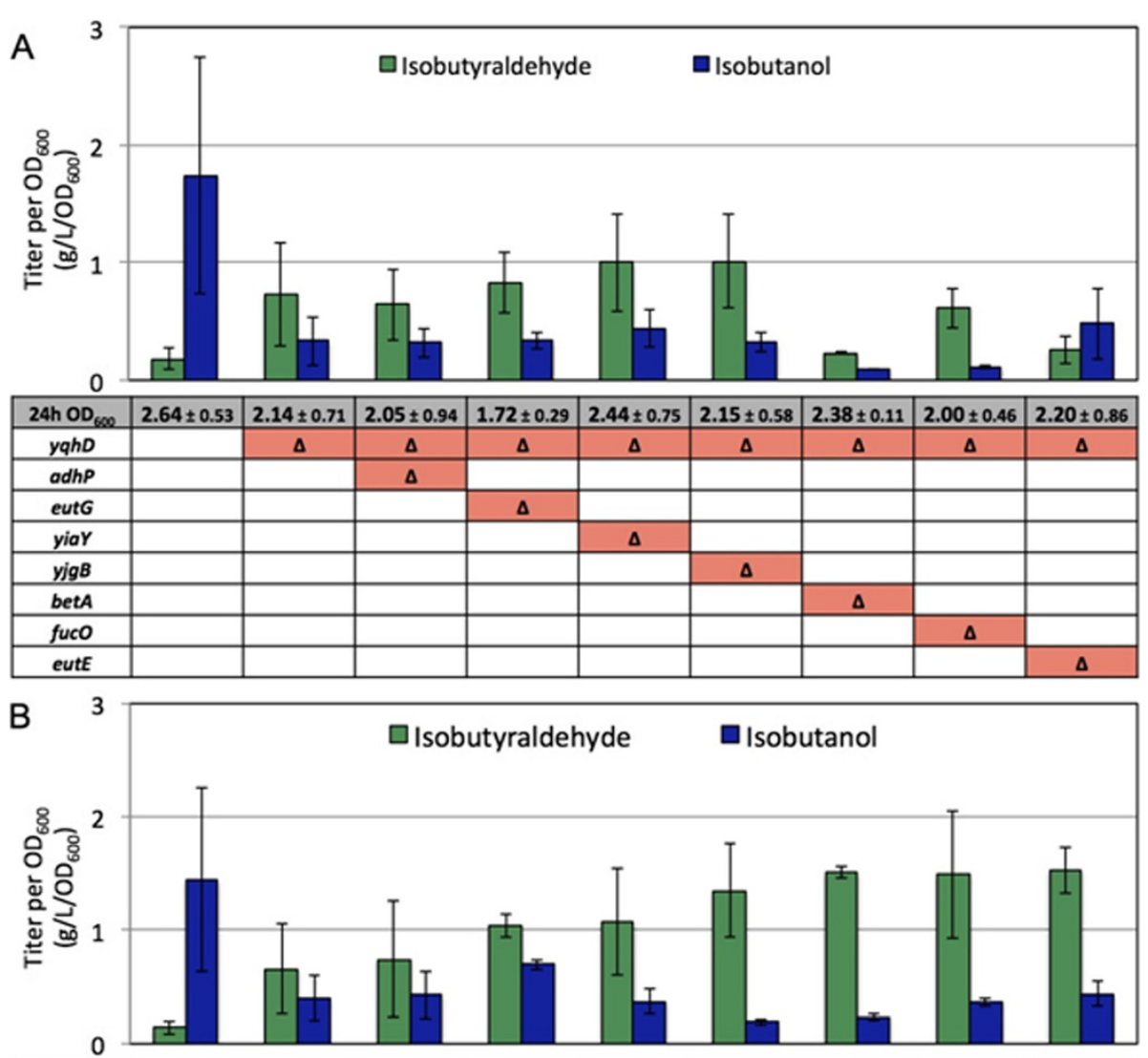

\begin{tabular}{|c|c|c|c|c|c|c|c|c|c|}
\hline $24 \mathrm{~h} \mathrm{OD}$ cos & $2.70 \pm 0.79$ & $2.96 \pm 0.58$ & $3.07 \pm 0.58$ & $3.24 \pm 0.26$ & $2.81 \pm 0.77$ & $3.06 \pm 0.39$ & $3.19 \pm 0.09$ & $1.68 \pm 0.22$ & $1.95 \pm 0.21$ \\
\hline yqhD & & $\Delta$ & $\Delta$ & $\Delta$ & $\Delta$ & $\Delta$ & $\Delta$ & $\Delta$ & $\Delta$ \\
\hline adhP & & & $\Delta$ & $\Delta$ & $\Delta$ & $\Delta$ & $\Delta$ & $\Delta$ & $\Delta$ \\
\hline eutG & & & & $\Delta$ & $\Delta$ & $\Delta$ & $\Delta$ & $\Delta$ & $\Delta$ \\
\hline yiar & & & & & $\Delta$ & $\Delta$ & $\Delta$ & $\Delta$ & $\Delta$ \\
\hline yjgB & & & & & & $\Delta$ & $\Delta$ & $\Delta$ & $\Delta$ \\
\hline betA & & & & & & & $\Delta$ & $\Delta$ & $\Delta$ \\
\hline fucO & & & & & & & & $\Delta$ & $\Delta$ \\
\hline eutE & & & & & & & & & $\Delta$ \\
\hline
\end{tabular}

Figure 2 Effects of individual and combined deletions of aldehyde reductases. Cells were grown at $37^{\circ} \mathrm{C}$ for $24 \mathrm{~h}$. " $\Delta$ " indicates gene deletion. All strains contained pGR03 (alsS, ilvC, and ilvD) and pSA129 (kivd). Titers represented as concentration per $\mathrm{OD}_{600}$ to adjust for variations in growth. Error bars represent the standard deviation of triplicate experiments.

a significant increase in isobutyraldehyde production of $1.35 \mathrm{~g} / \mathrm{L} / \mathrm{OD}_{600}$. Subsequent deletions of $\Delta$ bet $A, \Delta$ fucO, and $\triangle e u t E$ on the strain including $\triangle y q h D \triangle a d h P \triangle e u t G$ $\triangle y i a Y$ and $\triangle y j g B$ showed no additional reduction in isobutanol formation. Most strains showed similar growth after 24 hours to $\mathrm{OD}_{600} \sim 3$, except for the last two deletions $(\triangle f u c O$ and $\triangle e u t E$ ) which showed a marked decrease in 24 hour growth $\left(\mathrm{OD}_{600} \sim 1.7-2\right)$ (Figure $\left.2 \mathrm{~B}\right)$. The marked change in aldehyde ratio from 1:10 (JCL260) to as much as 7:1 (AL329( $\Delta y q h D \Delta a d h P \Delta e u t G \Delta y i a Y \Delta y j g B)$ ) represents a roughly $\sim 10$ fold increase in isobutyraldehyde production and $\sim 7.5$ fold decrease in isobutanol production (Figure 2B).

These results indicate that the remaining isobutanol production after deletion of $y q h D$ was the result of multiple enzymes that have IBR activity. Additionally, despite deleting our entire list of $I B R$ candidates in $E$. coli, there remains a small amount of isobutanol production. This suggests yet other enzymes are present that have IBR activity. Since no additional gene candidates were obvious in the $E$. coli genome and additional deletions became more difficult due to too many FLP recognition target sites [29], we did not search for further IBR candidates.

Overexpression of candidate IBRs to confirm IBR activities In order to determine conclusively which of the candidate IBRs were active toward isobutyraldehyde, we took the low isobutanol producing strain AL626 ( $\Delta y q h D$ $\Delta a d h P \quad \Delta e u t G \quad \Delta y i a Y \quad \Delta y j g B \quad \Delta$ betA $\Delta$ fucO) and 
overexpressed each candidate IBR in addition to the isobutyraldehyde pathway. In this way, if the candidate IBRs are active, overexpression of these would reverse the ratio of isobutyraldehyde to isobutanol to levels similar to that of the parent strain (JCL260). The candidate IBRs were individually cloned onto pSA138, downstream of kivd, by replacing yqhD using SLIC (see Methods). The new plasmids along with pGR03 were then introduced into AL626. Production from the strains overexpressing adhP, eutG, yjgB, or fucO led to a reversal of production, while the other enzymes did not, indicating that these have IBR activity (Figure 3a). To verify proper expression of each enzyme from plasmid, cell extracts were ran on SDS-PAGE. All protein expressions were confirmed. The strain overexpressing adhP or eutG produced $\sim 3 \mathrm{~g} / \mathrm{L} / \mathrm{OD}_{600}$, more than the strain overexpressing $y q h D\left(1.7 \mathrm{~g} / \mathrm{L} / \mathrm{OD}_{600}\right)$. This is likely because AdhP and EutG are NADH dependent [20,21,23], whereas YqhD is NADPH dependent [30]. The strain overexpressing fucO also produced slightly more than the strain overexpressing $y q h D\left(2 \mathrm{~g} / \mathrm{L} / \mathrm{OD}_{600}\right.$ vs $\left.1.7 \mathrm{~g} / \mathrm{L} / \mathrm{OD}_{600}\right)$, and is also NADH-dependent [25]. Since glycolysis produces two NADHs per one glucose, availability of $\mathrm{NADH}$ is generally higher in $E$. coli than NADPH. The strain overexpressing $y j g B$ produced similar amounts of isobutanol as the strain overexpressing $y q h D$, verifying its putative annotation of aldehyde reductase/alcohol dehydrogenase [26] to be correct. The reason that deletion of $y q h D$ has a greater affect than the other genes is likely due to higher expression levels from the genome. A comparison of mRNA levels from a transcriptome study show that mRNA levels of $y q h D$ are between 2 and 10 fold higher than the other candidate genes, except for betA [31]. It is also known that the expression of $y q h D$ is upregulated in the presence of aldehyde [32].

To further explore the activity of each IBR, enzyme assays were performed with each IBR candidate. We took the low isobutanol producing strain AL626 ( $\Delta y q h D$ $\triangle a d h P \Delta$ eutG $\triangle y i a Y \Delta y j g B \Delta$ betA $\Delta$ fucO) and overexpressed each candidate IBR. The candidate IBRs were individually cloned onto pZE12-luc, by replacing luc using SLIC (see Methods). The new plasmids and pZE12-luc (negative control) were then introduced into AL626. We assessed the activity of each enzyme with acetaldehyde (AA) and isobutyraldehyde (IBA) as substrates as well as with both cofactors, NADH and NADPH (Figure 3b). We confirmed aldehyde reductase activity of AdhP, EutG, $\mathrm{YjgB}$, and FucO with both AA and IBA as substrates. The active enzymes showed similar preference for AA and IBA when assayed with their preferred cofactor. AdhP was able to utilize both NADH and NADPH, but IBR activity was 2-fold higher with NADH. FucO showed about

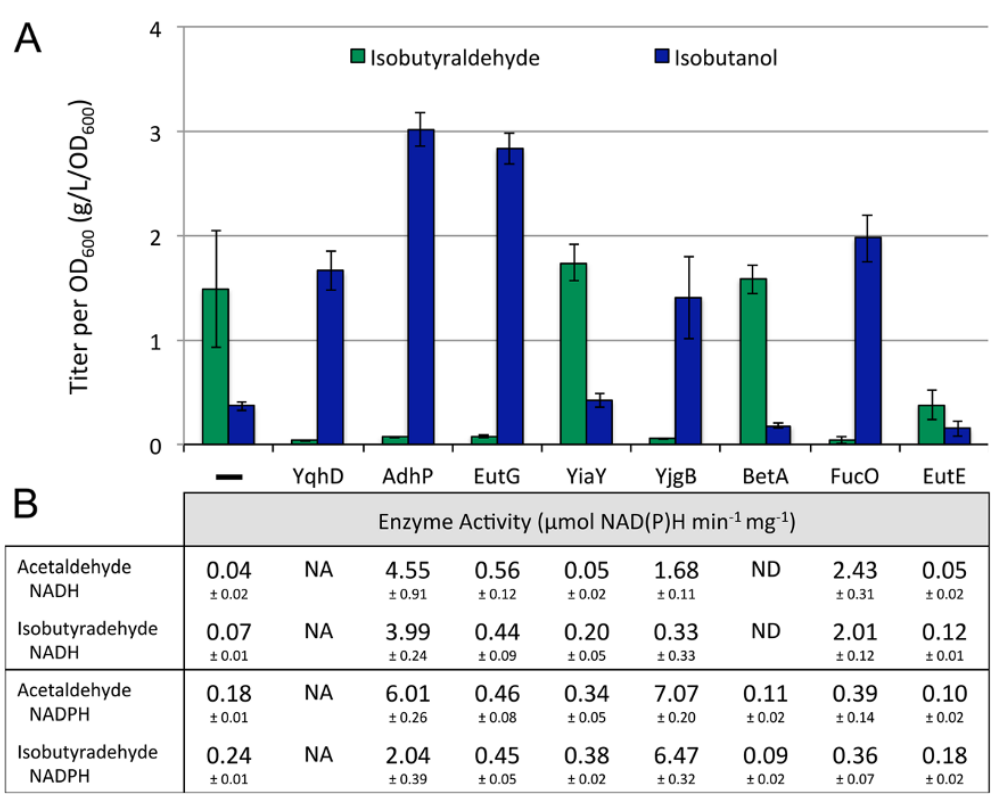

Figure 3 Overexpression of each candidate IBR in AL626. (A) Each candidate gene was cloned onto individual plasmids downstream of kivd

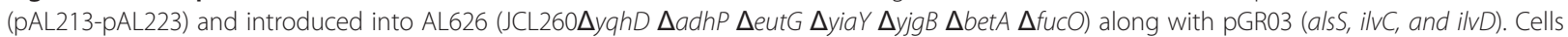
were grown at $37^{\circ} \mathrm{C}$ for $24 \mathrm{~h}$. Titers represented as concentration per $\mathrm{OD}_{600}$ to adjust for variations in growth. (B) Each gene was cloned onto individual plasmids and introduced into AL626. Cell extracts were assayed with acetaldehyde and isobutyraldehyde as substrates as well as with both cofactors (NADH \& NADPH). Enzyme activity is defined as $\mu$ mol NAD(P)H consumed per minute per mg of protein. $N A D(P) H$ consumption measured at $340 \mathrm{~nm}$. Error values represent the standard deviation of triplicate experiments. NA: not assayed. ND: not detectable. 


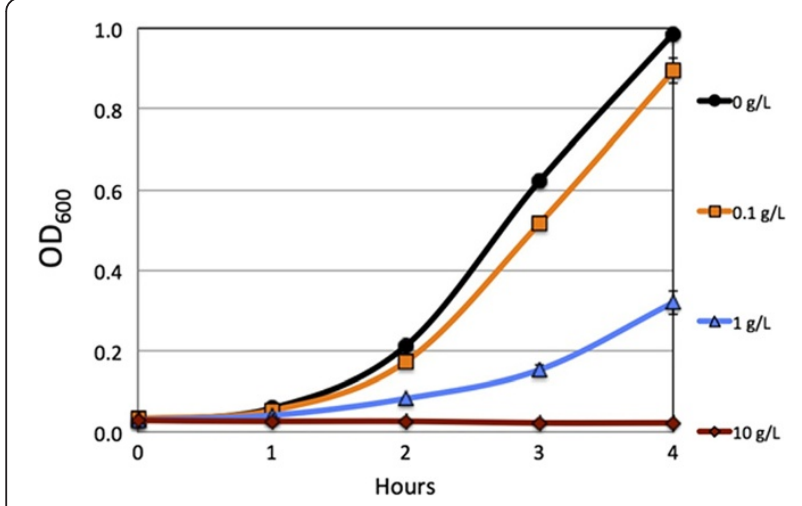

Figure 4 Comparison of growth with isobutyraldehyde stress. Time courses for the growth of E. coli strain AL626 (JCL260 $\Delta y$ ghD $\Delta a d h P \Delta$ eutG $\Delta$ yiar $\Delta y j g B \Delta$ betA $\Delta f u c O)$ in the presence of 0 (black), 0.1 (orange), 1 (blue), and $10 \mathrm{~g} / \mathrm{L}$ (red) isobutyraldehyde in $15 \mathrm{ml}$ screw-cap tubes. Optical density (OD) measurements taken every hour for 4 hours at $600 \mathrm{~nm}$. Error bars represent the standard deviation of triplicate experiments.

$50 \%$ the activity of AdhP, consistent with production levels from Figure 3a. FucO showed clear preference for $\mathrm{NADH}$, having $~ 6$-fold higher activity with NADH than with NADPH for both aldehydes. The activity of EutG which is a Fe-containing $\mathrm{ADH}$, as its sequence suggests [23], was not as high as compared to AdhP with AA $\left(0.56 \mu \mathrm{mol}\right.$ NADH $\left.\mathrm{min}^{-1} \mathrm{mg}^{-1}\right)$ and IBA $(0.44 \mu \mathrm{mol}$ NADH $\min ^{-1} \mathrm{mg}^{-1}$ ), but these activities were still 6 to 10fold greater compared to the negative control. YjgB preferred NADPH and showed the highest activity of all the enzymes with both AA $\left(7.07 \mu \mathrm{mol}\right.$ NADPH $\left.\mathrm{min}^{-1} \mathrm{mg}^{-1}\right)$ and IBA $\left(6.47 \mu \mathrm{mol} \mathrm{NADPH} \mathrm{min}^{-1} \mathrm{mg}^{-1}\right.$ ). YiaY, BetA, and EutE showed very weak or no aldehyde reductase activity with both AA and IBA. The absence of activity from YiaY and BetA with AA and IBA is notable since they were previously used to produce $>\mathrm{C} 5$ alcohols [24].

\section{Long-term production with in situ product removal}

With a couple of high isobutyraldehyde producing strains, we explored the feasibility of long-term production of isobutyraldehyde. From toxicity experiments (Figure 4), we observe no growth at $10 \mathrm{~g} / \mathrm{L}$ and significant growth inhibition with $1 \mathrm{~g} / \mathrm{L}$. Thus, we expect that toxicity of isobutyraldehyde accumulation could significantly hinder long-term production. In order to prevent inhibitory levels of aldehyde from accumulating, we applied a gas-stripping system to remove product in situ (Figure 5) [33]. Removal of product from a culture solution may also create a driving force for further product formation [34]. Furthermore, the redox balance of this pathway also necessitates sufficient supply of oxygen to the culture. Elimination of isobutanol formation negatively impacts the redox balance of the pathway under anaerobic conditions. Isobutanol formation results in a balanced redox state (Figure 1). As a result, isobutanol can be produced under anaerobic conditions. The isobutyraldehyde pathway, however, yields an excess of 1 NADH per glucose. This excess necessitates aerobic conditions in order to recycle the $\mathrm{NAD}^{+}$pool through oxidative phosphorylation. Thus, using a gas-stripping system provides the cells with ample oxygen while simultaneously removing isobutyraldehyde from the culture.

In this set up, air flows through the culture flask, where vaporized isobutyraldehyde is captured in a series of cold $\left(\sim 4^{\circ} \mathrm{C}\right)$ condensers and 'trap' bottles. The high volatility of isobutyraldehyde $\left(172 \mathrm{mmHg}\right.$ at $\left.25^{\circ} \mathrm{C}\right)$ facilitates this process [14]. Since isobutyraldehyde is known to catalytically react with oxygen in the air between $30-50^{\circ} \mathrm{C}$ and convert to isobutyric acid [35], we conducted production at $30^{\circ} \mathrm{C}$ to minimize this. Furthermore, production of isobutanol at $30^{\circ} \mathrm{C}$ has been performed with greater success than at $37^{\circ} \mathrm{C}$ [34].

Based on the results from Figure 3, we used AL626 for long-term production experiments (Figure 6). Production

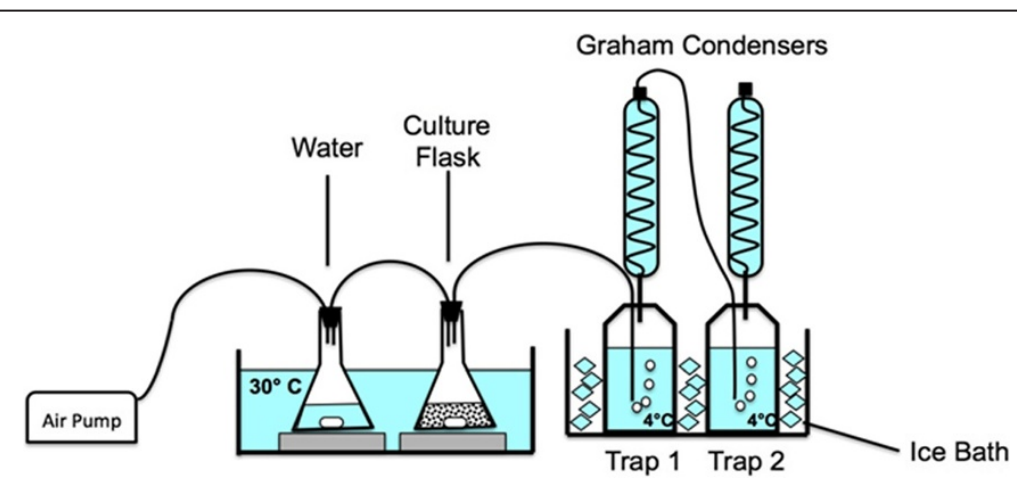

Figure $\mathbf{5}$ Schematic of gas stripping system. Sterile air is pumped $(3 \mathrm{cc} / \mathrm{min})$ into a flask containing $100 \mathrm{~mL}$ sterile water to saturate the air and thereby prevent evaporation of water in the culture flask. Vaporized product (isobutyraldehyde) is then carried into a series of traps and condensers held at $1-4{ }^{\circ} \mathrm{C}$ where it is captured for quantification. Removal of product during production greatly reduces accumulation which is often toxic to the cells. 
was carried out in a $250 \mathrm{~mL}$ baffled shake-flask under constant stirring. Cultures were initially grown at $37^{\circ} \mathrm{C}$ for faster growth and induced at $\mathrm{OD}_{600} \sim 0.4$. Upon induction, the temperature of the culture bath was quickly brought down to $30^{\circ} \mathrm{C}$. We optimized the airflow rate $(3 \mathrm{cc} / \mathrm{min})$ of the system such that the majority of isobutyraldehyde was collected in the first trap bottle and with minimal amounts remaining in the culture flask. Every 24 hours, $10 \%$ of the culture was removed for growth,
$\mathrm{pH}$, and product analysis and replaced with fresh media containing $\sim 370 \mathrm{~g} / \mathrm{L}$ glucose. Trap bottles were also analyzed and replaced every 24 hours with new trap bottles.

In the first 24 and 48 hours of production, the strain averaged $8 \mathrm{~g} / \mathrm{L} /$ day of isobutyraldehyde (Figure 6B) with low isobutanol formation (0.7-1.3 g/L/day). As production continued from 72 to 120 hours, we observed a consistent decline of isobutyraldehyde productivity from $8 \mathrm{~g} / \mathrm{L} /$ day to an eventual $5 \mathrm{~g} / \mathrm{L} /$ day. This notably corresponded with a
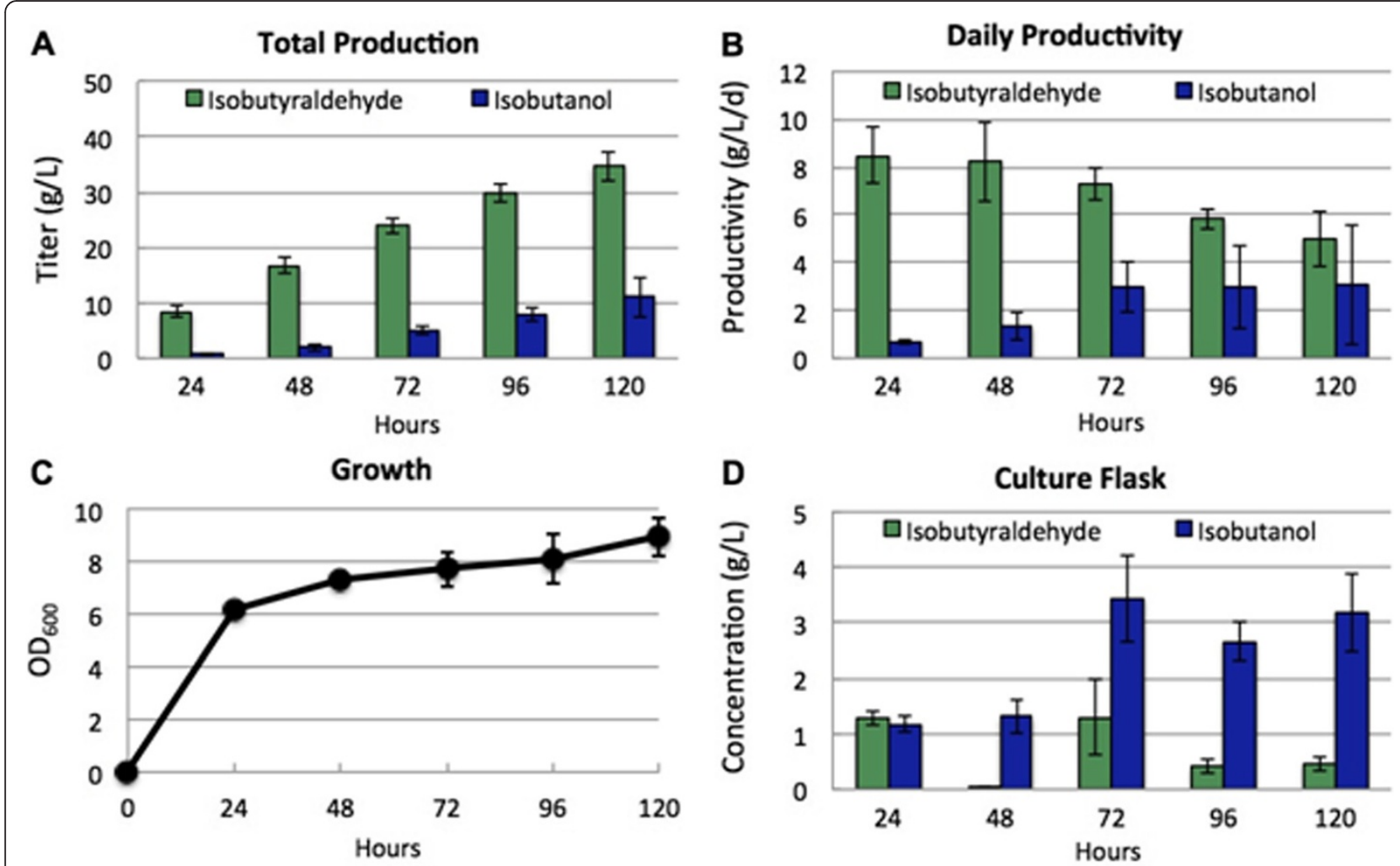

D

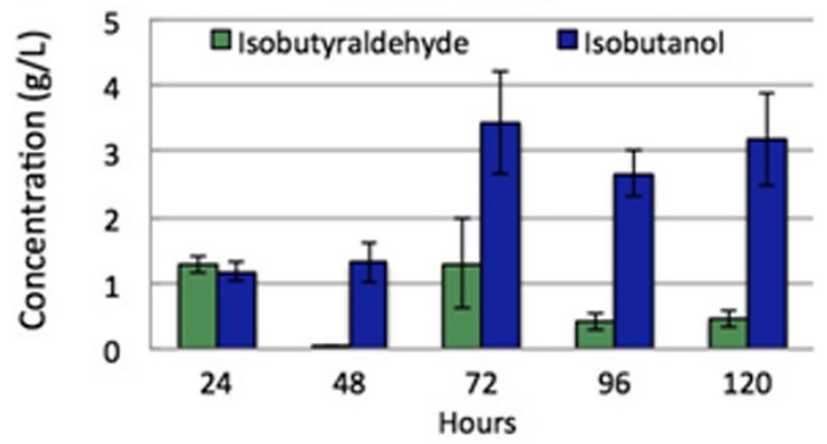

$\mathbf{E}$

$\mathbf{F}$

pH
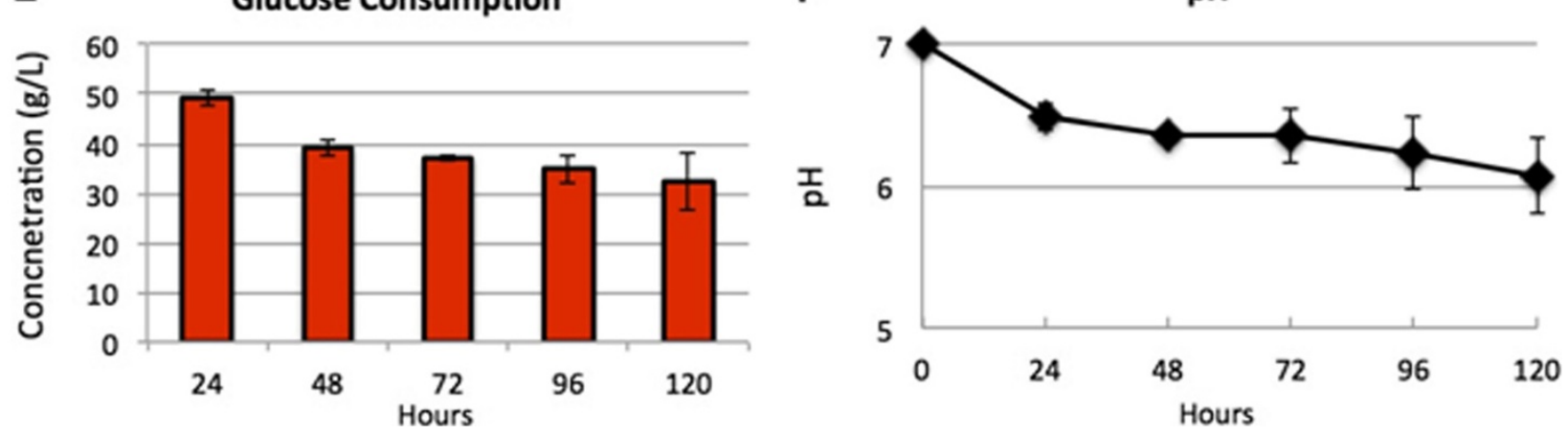

Figure 6 Long-term production of isobutyraldehyde with a gas stripping system. (A) Total accumulated production of isobutyraldehyde (green) and isobutanol (blue). (B) Daily productivity of isobutyraldehyde (green) and isobutanol (blue). (C) Time profiles of cell growth. (D) Concentrations of isobutyraldehyde (green) and isobutanol (blue) in the production culture to determine effectiveness of product removal by the system. (E) Daily glucose consumption of the culture. (F) Time profile of pH of the production culture. pGR03 (alsS, ilvC, and ilvD) and pSA129 (kivd) were introduced to AL626. Cells were inoculated 1\% (vol/vol) from the overnight culture and grown in $100 \mathrm{~mL}$ production media (M9 media containing $5 \mathrm{~g} / \mathrm{L}$ yeast extract and $50 \mathrm{~g} / \mathrm{L}$ glucose). Every 24 hours, 10\% of culture was removed for analysis, and replaced with production media containing $370 \mathrm{~g} / \mathrm{L}$ glucose (dilutes to $37 \mathrm{~g} / \mathrm{L} / \mathrm{d}$ ). Error bars represent the standard deviation of triplicate experiments. 
similar decline in glucose consumption from $\sim 37 \mathrm{~g} / \mathrm{L} /$ day at 48 hours, down to $32 \mathrm{~g} / \mathrm{L} /$ day at 120 hours (Figure 6F). Surprisingly, we observed a marked increase in daily isobutanol formation beginning at 72 hours. After 120 hours, production of isobutanol began to overtake that of isobutyraldehyde in some trials, suggesting an up regulation of one or more unknown IBRs. For this reason, we stopped production after 120 hours. The culture grew to $\mathrm{OD}_{600}$ 6.2 after the first day and increased slowly thereafter up to $\mathrm{OD}_{600} 9$ after 120 hours (Figure 6C). This suggests the culture received sufficient oxygen to achieve redox balance throughout the production. The $\mathrm{pH}$ of the culture (Figure 6F) remained fairly stable throughout production, decreasing only by 1 unit to a $\mathrm{pH}$ of about 6 after 120 hours. In the future, maintaining $\mathrm{pH}$ at 7 by addition of base or by using a buffer may improve yield and culture stability with this system.

Overall, isobutyraldehyde levels in the culture flask remained low, typically between $0.5 \mathrm{~g} / \mathrm{L}$ and $1.5 \mathrm{~g} / \mathrm{L}$ (Figure 6D). The stable growth and relatively low product accumulation (Figure 6D), indicates the gasstripping system was successful in reducing the potential toxicity of isobutyraldehyde. Development of an isobutyraldehyde tolerant strain may improve cell growth and culture stability during production, possibly circumventing the need for gas-stripping. Strains tolerant of isobutanol have previously been developed for similar reasons [36]. However, an increase in isobutanol tolerance did not correlate with improved production [34]. Our strain's productivity of $8 \mathrm{~g} / \mathrm{L} /$ day is roughly 8 -fold greater than toxic levels of isobutyraldehyde $(\sim 1 \mathrm{~g} / \mathrm{L})$. Achieving a strain that can tolerate these titers may be difficult to develop. Additionally, utilizing gas-stripping has the added advantage of purifying isobutyraldehyde during production, which may be a cost saving method under industrial scale production.

Isobutyraldehyde production from this strain totaled $35 \mathrm{~g} / \mathrm{L}$ after 120 hours (Figure 6A). Including formation of isobutanol $(\sim 10 \mathrm{~g} / \mathrm{L})$, total production reached $\sim 45 \mathrm{~g} /$ L. The theoretical molar yield of isobutyraldehyde from glucose is $1: 1$. This results in a gram per gram yield of $0.40 \mathrm{~g}$ isobutyraldehyde/g glucose. In the first 48 hours of production, we achieved a yield of $47 \%$ of the theoretical maximum. The yield remained relatively constant throughout the experiment with a final yield of $45 \%$ of the theoretical maximum after 120 hours.

The reason for the large increase in isobutanol formation is not entirely obvious. Growth, $\mathrm{pH}$, and overall yield remained stable throughout the experiment, but glucose consumption and isobutyraldehyde production slowly decreased. Despite the discovery and removal of five IBRs from the genome, still further significant IBR activity exists in our E. coli strain. One possibility is that expression of the unknown enzyme(s) is low early on in production and later upregulated. Cells that are able to increase expression of such enzyme(s) will likely experience lower toxicity and may begin to outgrow other cells within the culture. Thus, further exploration and elimination of this IBR activity will be needed to achieve more stable isobutyraldehyde production after 72 hours.

\section{Conclusions}

In this work, we demonstrated the renewable production of isobutyraldehyde, an important chemical feedstock, from E. coli. We identified AdhP, EutG, YjgB, and FucO as isobutyraldehyde reductases, while YiaY, BetA, and EutE were not active toward isobutyraldehyde. With in situ product removal, we achieved a total isobutyraldehyde production of $35 \mathrm{~g} / \mathrm{L}$ after 5 days. This industrially relevant titer represents a yield of $45 \%$ of the theoretical maximum. However, after the second day, isobutanol formation began to increase markedly, suggesting upregulation of yet additional $\operatorname{IBR}(\mathrm{s})$ in E. coli.

The lingering presence of isobutanol indicates that additional IBRs still exist in $E$. coli which we have yet to identify. In this work, we evaluated genes more specifically annotated or characterized as alcohol dehydrogenases. It is possible that other non-specific oxidoreductases are contributing to IBR activity in the cell. E. coli contains more than a dozen other putative, less specifically annotated, NAD $(\mathrm{P}) \mathrm{H}$ dependent oxidoreductases. Going forward, applying transcriptome analysis, bioinformatics, and other methods may uncover the remaining IBR(s). Further exploration and elimination of these reductases will be required to improve the long-term production stability of this strain.

\section{Methods \\ Reagents}

Rapid Ligation Kit was purchased from Roche. All other enzymes were purchased from New England Biolabs (Ipswich, MA). All synthetic oligonucleotides were ordered from Integrated DNA Technologies (Coralville, IA). DNA sequencing services were done by Davis Sequencing (Davis, CA). Zymo DNA Clean and Concentrator kit and Gel Recovery kit (Zymo Research, Irvine, CA) were used to purify all PCR products. Isobutanol and Isobutyraldehyde were purchased from Sigma Aldrich (St. Louis, MO). 1-pentanol was purchased from Acros Organics (Belgium).

\section{Media}

Overnight cultures were grown in $5 \mathrm{~mL}$ Luria Broth (LB) containing appropriate antibiotics. Antibiotic concentrations were as follows: Kanamycin $(50 \mu \mathrm{g} / \mathrm{mL})$, Chloramphenicol $(40 \mu \mathrm{g} / \mathrm{mL})$, Ampicillin $100(\mu \mathrm{g} / \mathrm{mL})$, 
Tetracycline $(20 \mu \mathrm{g} / \mathrm{mL})$. Production was carried out with M9 medium containing $5 \mathrm{~g} / \mathrm{L}$ yeast extract, $\sim 50 \mathrm{~g} / \mathrm{L}$ glucose, and 1000-fold dilution of A5 trace metal mix (2.86 $\mathrm{g} \mathrm{H}_{3} \mathrm{BO}_{3}, 1.81 \mathrm{~g} \mathrm{MnCl}_{2} \cdot 4 \mathrm{H}_{2} \mathrm{O}, 0.222 \mathrm{~g} \mathrm{ZnSO}$ $.7 \mathrm{H}_{2} \mathrm{O}, 0.39 \mathrm{~g} \mathrm{Na}_{2} \mathrm{MoO}_{4} \cdot 2 \mathrm{H}_{2} \mathrm{O}, 0.079 \mathrm{~g} \mathrm{CuSO}_{4} \cdot 5 \mathrm{H}_{2} \mathrm{O}$, $49.4 \mathrm{mg} \mathrm{Co}\left(\mathrm{NO}_{3}\right)_{2} \cdot 6 \mathrm{H}_{2} \mathrm{O}$ per liter water $)$.

\section{Gene deletions}

All gene deletions were carried out by P1 transduction [37] with the aid of strains from the Keio collection [29], except for eutE. To delete eutE, the method developed by Datsenko and Wanner [27] was used due to its proximity to eutG. The deleted fragments were verified by PCR and sequencing. All strains used in this study are listed in Table 1.

\section{Plasmid construction, cloning, and transformations}

To construct pGR03, first pSA69 [5] was digested with AatII and SacI, and treated with Antarctic phosphatase. Then the chloramphenicol resistance gene was taken from pZA31-luc [28] by digestion with AatII and SacI. The two fragments were gel purified, ligated, and introduced into E. coli XL-1 blue strain.

All other plasmids (pAL156-158, pAL162, pAL213215, pAL217-223) were constructed using sequence and ligation-independent cloning (SLIC) [38]. In general, primers for inserts (GR147-154, GR188-193, GR219GR232) were designed with $\sim 25$ bp priming to the target gene and a 20-25 bp 'linker' to target vector. All candidate IBRs were amplified by PCR from E. coli JCL16 genome DNA. Plasmids were verified by colony PCR, by digestion with restriction enzymes, and by sequencing. All plasmids and oligonucleotides are listed in Table 1 and Table 2, respectively.

Vector was amplified from pSA138 with GR180 and GR181, and digested with DpnI to reduce background associated from plasmid template. Primers (GR219 and GR221 for adhP, GR221 and GR222 for eutG, GR223 and GR224 for yiaY, GR225 and GR226 for yjgB, GR227 and GR228 for betA, GR229 and GR230 for fucO, and GR231 and GR232 for eutE) were used to amplify each gene from E. coli JCL16 genome DNA and cloned with SLIC onto the pSA138 vector fragment.

Vector was amplified from pZE12-luc with GR145 and GR146, and digested with DpnI to reduce background associated from plasmid template. Primers (GR147 and GR148 for adhP, GR149 and GR150 for eutG, GR151 and GR152 for $y i a Y$, GR153 and GR154 for $y j g B$, GR188 and GR189 for betA, GR190 and GR191 for fucO, and GR192 and GR193 for eutE) were used to amplify each gene from E. coli JCL16 genome DNA and cloned with SLIC onto the pZE12-luc vector fragment.
Table 2 Primer sequences

\begin{tabular}{|c|c|}
\hline Primer & Sequence $5^{\prime} \rightarrow 3^{\prime}$ \\
\hline GR180 & TCTAGAGGCATCAAATAAAACGAAAGGCTC \\
\hline GR181 & GGTATATCTCCTGCATGCTTATGATTTATTTG \\
\hline GR219 & GCATGCAGGAGAAAGGTCACatgaaggctgcagttgttacgaagg \\
\hline GR220 & TITATTTGATGCCTCTAGAttagtgacggaaatcaatcaccatg \\
\hline GR221 & GCATGCAGGAGAAAGGTCACatgcaaaatgaattgcagaccgcgc \\
\hline GR222 & TITATTTGATGCCTCTAGAttattgcgccgctgcgtacaggecg \\
\hline GR223 & GCATGCAGGAGAAAGGTCACatgg cagcttcaacgttctttattc \\
\hline GR224 & TTTATTTGATGCCTCTAGAttacatcgctgcgcgataaatcgcc \\
\hline GR225 & GCATGCAGGAGAAAGGTCACatgtcgatgataaaaagctatgccg \\
\hline GR226 & TITATTTGATGCCTCTAGAtcaaaaatcggctttcaacaccacg \\
\hline GR227 & GCATGCAGGAGAAAGGTCACAtgcaatttgactacatcattattg \\
\hline GR228 & TITATTTGATGCCTCTAGAtcattttttcgctctcaccggcatc \\
\hline GR229 & GCATGCAGGAGAAAGGTCACatgatggctaacagaatgattctga \\
\hline GR230 & TITATTGATGCCTCTAGAttaccaggcggtatggtaaagctct \\
\hline GR231 & GCATGCAGGAGAAAGGTCACatgaatcaacaggatattgaacagg \\
\hline GR232 & TITATTTGATGCCTCTAGAttaaacaatgcgaaacgcatcgact \\
\hline GR145 & GGTACCTTTCTCCTCTITAATGAA \\
\hline GR146 & TAATGACTCTAGAGGCATCAAATAA \\
\hline GR147 & ATTCATTAAAGAGGAGAAAGGTACCatgaaggctgcagttgttacgaagg \\
\hline GR148 & TTATTTGATGCCTCTAGAGTCATTAttagtgacggaaatcaatcaccatg \\
\hline GR149 & ATTCATTAAAGAGGAGAAAGGTACCatgcaaaatgaattgcagaccgcgc \\
\hline GR150 & TTATTTGATGCCTCTAGAGTCATTAttattgcgccgctgcgtacaggccg \\
\hline GR151 & ATTCATTAAAGAGGAGAAAGGTACCatggcagcttcaacgttctttattc \\
\hline GR152 & TTATTTGATGCCTCTAGAGTCATTAttacatcgctgcgcgataaatcgcc \\
\hline GR153 & ATTCATTAAAGAGGAGAAAGGTACCatgtcgatgataaaaagctatgccg \\
\hline GR154 & TTATTTGATGCCTCTAGAGTCATTAtcaaaaatcggctttcaacaccacg \\
\hline GR188 & ATTCATTAAAGAGGAGAAAGGTACCAtgcaatttgactacatcattattggtg \\
\hline GR189 & TAATTGATGCCTCTAGAGTCATTAtcattttttcgctctcaccggcatc \\
\hline GR190 & ATTCATTAAAGAGGAGAAAGGTACCatgatggctaacagaatgattctgaac \\
\hline GR191 & TTATTTGATGCCTCTAGAGTCATTAttaccaggcggtatggtaaagctc \\
\hline GR192 & ATTCATTAAAGAGGAGAAAGGTACCatgaatcaacaggatattgaacaggtg \\
\hline GR193 & TTATTTGATGCCTCTAGAGTCATTAttaaacaatgcgaaacgcatcgact \\
\hline
\end{tabular}

\section{Screw-cap tube production}

For isobutyraldehyde production test, $1 \%(\mathrm{vol} / \mathrm{vol})$ of the overnight culture was inoculated in $5 \mathrm{~mL}$ production media in $15 \mathrm{~mL}$ screw-cap culture tubes and grown at $37^{\circ} \mathrm{C}$ in a rotary shaker $(250 \mathrm{RPM})$ until $\mathrm{OD}_{600} \sim 0.4$, then induced with $1 \mathrm{mM}$ isopropyl- $\beta$-D-thio-galactoside (IPTG) and allowed to produce for 24 hours after induction. Screw-cap tubes were tightly sealed to prevent evaporation of isobutyraldehyde.

\section{Aldehyde reductase activity assay}

The plasmids pZE12-luc, pAL162, pAL156-158, and pAL213-214 were introduced into AL626. The strains 
were grown to $\mathrm{OD}_{600}$ value of $\sim 0.4$ in $5 \mathrm{~mL} \mathrm{LB}$ medium at $37^{\circ} \mathrm{C}$, followed by adding $1 \mathrm{mM}$ IPTG. Protein overexpression was performed at $37^{\circ} \mathrm{C}$ for $4 \mathrm{~h}$. Then $1.8 \mathrm{~mL}$ of cells were centrifuged at 13,000 RPM for 10 minutes, resuspended in $300 \mu \mathrm{L}$ BugBuster Protein Extraction Reagent (Novagen, San Diego, CA, USA), and incubated at room temperature for $20 \mathrm{~min}$ for cell lysis. The samples were centrifuged for $20 \mathrm{~min}, 16,000 \mathrm{~g}$, at $4^{\circ} \mathrm{C}$. Supernatants were taken for enzyme assays. ADH activities were measured by following the reduction of acetaldehyde or isobutyraldehyde with NADH or NADPH at $340 \mathrm{~nm}$ at $37^{\circ} \mathrm{C}$ using a Synergy H1 Hybrid Plate Reader from BioTek Instruments, Inc. (Winooski, VT). The assay mixture contained $25 \mathrm{mM}$ acetaldehyde or isobutyraldehyde, $50 \mathrm{mM} 3$-( $N$-morpholino)propanesulfonic acid (MOPS) buffer (pH 7.0), $0.2 \mathrm{mM}$ Tris- $\mathrm{Cl}$ (pH 7.00), $0.2 \mathrm{mM}$ $\mathrm{NAD}(\mathrm{P}) \mathrm{H}$, and $12.5 \mathrm{mM}$ potassium phosphate buffer $(\mathrm{pH}$ 7.5). One unit of activity is defined as the oxidation of $1 \mu \mathrm{mol}$ of $\mathrm{NAD}(\mathrm{P}) \mathrm{H}$ per minute per mg protein. Protein concentrations were measured using $5 \mathrm{x}$ Advanced Protein Assay Reagent (Cytoskeleton Inc., Denver, CO) by diluting $5 \mu \mathrm{L}$ of cell extract in $1 \mathrm{~mL}$ of $1 \mathrm{x}$ Advanced Protein Assay Reagent and measuring the $\mathrm{OD}_{590}$ of the mixture. Bovine Serum Albumin (BSA) from NEB was used to prepare a standard curve.

\section{Gas stripping apparatus}

Rubber stoppers were used to cap the culture flask and water flask. Two holes were made in each rubber stopper where small, customized, metal pipes were inserted. All flasks, traps, and condensers were connected with clear, flexible, chemical resistant, plastic tubing (Tygon tubing). Air flow rate was set to $3 \mathrm{cc} / \mathrm{min}$ with a flow regulator. Trap bottles were filled with $600 \mathrm{~mL}$ of water. Culture volume and water flask volume were both $100 \mathrm{~mL}$. Culture was initially grown at $37^{\circ} \mathrm{C}$ until $\mathrm{OD}_{600} \sim 0.4$, then $1 \mathrm{mM}$ IPTG was added and the temperature was quickly brought down to $30^{\circ} \mathrm{C}$ for the remainder of the experiment. When trap bottles were collected for samples, $200 \mathrm{~mL}$ water was washed down each condenser to rinse any product into the trap bottles. Final trap volume was determined with a $1000 \mathrm{~mL}$ graduated cylinder.

\section{GC analysis}

Concentrations of all products, except glucose, were analyzed by Gas Chromatography equipped with a flame ionization detector (FID). The GC system is a Shimadzu GC-2010 with an AOC-20 S auto sampler and AOC-20i Auto Injector. The column used was a DB-Wax capillary column (30 m length, 0.32-mm diameter, $0.50-\mu \mathrm{m}$ film thickness) from Agilent Technologies. GC oven temperature was initially held at $40^{\circ} \mathrm{C}$ for 3 minutes, then increased at a rate of $45^{\circ} \mathrm{C} \mathrm{min}^{-1}$ until $235^{\circ} \mathrm{C}$ and held for $3 \mathrm{~min}$.
Injector temperature was held at $225^{\circ} \mathrm{C}$ and FID detector was held at $330^{\circ} \mathrm{C}$. Injection volume was $0.5 \mu \mathrm{L}$, injected at a $15: 1$ split ratio. Helium was used as the carrier gas. 1-pentanol was used as internal standard.

\section{Glucose quantification and growth measurements}

Glucose was measured using a Sucrose, Fructose, and Glucose kit from Megazyme International Unlimited. Glucose assays and Optical densities (OD) were measured at $340 \mathrm{~nm}$ and $600 \mathrm{~nm}$, respectively, with a Genesis $10 \mathrm{~S}$ UV-vis Spectrophotometer (Thermo Scientific).

\section{Competing interests}

A provisional patent regarding this technology has been filed.

\section{Authors' contributions}

S.A. and G.M.R designed experiments; G.M.R. performed the experiments; S.A. and G.M.R. analyzed the data; and S.A. and G.M.R. wrote the paper. All

authors read and approved the final manuscript.

\section{Acknowledgements}

This work was supported by University of California, Davis Startup Funding to SA. G.M.R. is supported by a Sloan fellowship. We are grateful to lara M.P. Machado, Tony Pan and Oliver G. Hernaez for experimental assistance.

Received: 14 April 2012 Accepted: 3 June 2012

Published: 25 June 2012

\section{References}

1. McFarlane J, Robinson S: Survey of Alternative Feedstocks for Commodity Chemical Manufacturing:: Oak Ridge National Laboratory; 2007 [http://info. ornl.gov/sites/publications/files/Pub8760.pdf].

2. Keasling JD: Manufacturing molecules through metabolic engineering. Science 2010, 330:1355-1358.

3. Zhang F, Rodriguez S, Keasling JD: Metabolic engineering of microbial pathways for advanced biofuels production. Curr Opin Biotechnol 2011, 22:775-783.

4. Rodriguez GM, Atsumi S: Synthetic Approach to Produce C3-C6 Alcohols from Microorganisms. Curr Chem Biol 2012, 6:32-41.

5. Atsumi S, Hanai T, Liao JC: Non-fermentative pathways for synthesis of branched-chain higher alcohols as biofuels. Nature 2008, 451:86-89.

6. Nakamura CE, Whited GM: Metabolic engineering for the microbial production of 1,3-propanediol. Curr Opin Biotechnol 2003, 14:454-459.

7. Keasling JD: Synthetic biology for synthetic chemistry. ACS Chem Biol 2008, 3:64-76

8. Shanmugam KT, Ingram LO: Engineering biocatalysts for production of commodity chemicals. J Mol Microbiol Biotechnol 2008, 15:8-15.

9. Villela Filho M, Araujo C, Bonfa A, Porto W: Chemistry based on renewable raw materials: perspectives for a sugar cane-based biorefinery. Enzyme Res 2011 2011, 654596

10. Yim H, Haselbeck R, Niu W, Pujol-Baxley C, Burgard A, Boldt J, Khandurina J, Trawick JD, Osterhout RE, Stephen R, et al: Metabolic engineering of Escherichia coli for direct production of 1,4-butanediol. Nat Chem Biol 2011, 7:445-452.

11. Zhang K, Woodruff AP, Xiong M, Zhou J, Dhande YK: A synthetic metabolic pathway for production of the platform chemical isobutyric acid. ChemsusChem 2011, 4:1068-1070.

12. Thakker C, Martinez I, San KY, Bennett GN: Succinate production in Escherichia coli. Biotechnol J 2012, 7:213-224.

13. Lee S, Kim B, Park K, Um Y, Lee J: Synthesis of Pure meso-2,3-Butanediol from Crude Glycerol Using an Engineered Metabolic Pathway in Escherichia coli. Appl Biochem Biotechnol 2012, 166:1801-1813.

14. SIDS Initial Assessment Report: ISOBUTANAL CAS N: 78-84-2.: Organization for Economic Cooperation and Development (OECD); 1996 [http://www. inchem.org/documents/sids/sids/78842.pdf].

15. Sentheshanmuganathan S, Elsden SR: The mechanism of the formation of tyrosol by Saccharomyces cerevisiae. Biochem J 1958, 69:210-218. 
16. Atsumi S, Wu TY, Eckl EM, Hawkins SD, Buelter T, Liao JC: Engineering the isobutanol biosynthetic pathway in Escherichia coli by comparison of three aldehyde reductase/alcohol dehydrogenase genes. App/ Microbiol Biotechnol 2010, 85:651-657.

17. Atsumi S, Cann AF, Connor MR, Shen CR, Smith KM, Brynildsen MP, Chou KJ, Hanai T, Liao JC: Metabolic engineering of Escherichia coli for 1-butanol production. Metab Eng 2008, 10:305-311.

18. Atsumi S, Higashide W, Liao JC: Direct photosynthetic recycling of carbon dioxide to isobutyraldehyde. Nat Biotechnol 2009, 27:1177-1180.

19. Keseler IM, Collado-Vides J, Santos-Zavaleta A, Peralta-Gil M, Gama-Castro S, Muniz-Rascado L, Bonavides-Martinez C, Paley S, Krummenacker M, Altman T, et al: EcoCyc: a comprehensive database of Escherichia coli biology. Nucleic Acids Res 2011, 39:D583-590.

20. Shafqat J, Hoog JO, Hjelmqvist L, Oppermann UC, Ibanez C, Jornvall H: An ethanol-inducible MDR ethanol dehydrogenase/acetaldehyde reductase in Escherichia coli: structural and enzymatic relationships to the eukaryotic protein forms. Eur J Biochem 1999, 263:305-311.

21. Penrod JT, Roth JR: Conserving a volatile metabolite: a role for carboxysome-like organelles in Salmonella enterica. J Bacteriol 2006, 188:2865-2874.

22. Brinsmade SR, Paldon T, Escalante-Semerena JC: Minimal functions and physiological conditions required for growth of Salmonella enterica on ethanolamine in the absence of the metabolosome. J Bacteriol 2005, 187:8039-8046.

23. Stojiljkovic I, Baumler AJ, Heffron F: Ethanolamine utilization in Salmonella typhimurium: nucleotide sequence, protein expression, and mutational analysis of the cchA cchB eutE eutJ eutG eutH gene cluster. J Bacteriol 1995, 177:1357-1366.

24. Dellomonaco C, Clomburg JM, Miller EN, Gonzalez R: Engineered reversal of the beta-oxidation cycle for the synthesis of fuels and chemicals. Nature 2011, 476:355-359.

25. Montella C, Bellsolell L, Perez-Luque R, Badia J, Baldoma L, Coll M, Aguilar J: Crystal structure of an iron-dependent group III dehydrogenase that interconverts L-lactaldehyde and L-1,2-propanediol in Escherichia coli. J Bacteriol 2005, 187:4957-4966

26. Riley M, Abe T, Arnaud MB, Berlyn MK, Blattner FR, Chaudhuri RR, Glasner JD, Horiuchi T, Keseler IM, Kosuge T, et al: Escherichia coli K-12: a cooperatively developed annotation snapshot-2005. Nucleic Acids Res 2006, 34:1-9.

27. Datsenko KA, Wanner BL: One-step inactivation of chromosomal genes in Escherichia coli K-12 using PCR products. Proc Natl Acad Sci U S A 2000, 97:6640-6645.

28. Lutz $\mathrm{R}$, Bujard $\mathrm{H}$ : Independent and tight regulation of transcriptional units in Escherichia coli via the LacR/O, the TetR/O and AraC/I1-I2 regulatory elements. Nucleic Acids Res 1997, 25:1203-1210.

29. Baba T, Ara T, Hasegawa M, Takai Y, Okumura Y, Baba M, Datsenko KA, Tomita M, Wanner BL, Mori H: Construction of Escherichia coli K-12 in-frame, single-gene knockout mutants: the Keio collection. Mol Syst Biol 2006, 2:E1-E11.

30. Sulzenbacher G, Alvarez K, Van Den Heuvel RH, Versluis C, Spinelli S, Campanacci V, Valencia C, Cambillau C, Eklund H, Tegoni M: Crystal structure of E.coli alcohol dehydrogenase YqhD: evidence of a covalently modified NADP coenzyme. J Mol Biol 2004, 342:489-502.

31. Shiroguchi $K$, Jia TZ, Sims PA, Xie XS: Digital RNA sequencing minimizes sequence-dependent bias and amplification noise with optimized single-molecule barcodes. Proc Natl Acad Sci U S A 2012, 109:1347-1352.

32. Jarboe LR: YqhD: a broad-substrate range aldehyde reductase with various applications in production of biorenewable fuels and chemicals. Appl Microbiol Biotechnol 2011, 89:249-257.

33. Inokuma K, Liao JC, Okamoto M, Hanai T: Improvement of isopropanol production by metabolically engineered Escherichia coli using gas stripping. J Biosci Bioeng 2010, 110:696-701.

34. Baez A, Cho KM, Liao JC: High-flux isobutanol production using engineered Escherichia coli: a bioreactor study with in situ product removal. Appl Microbiol Biotechnol 2011, 90:1681-1690.

35. Hagemeyer HJ, De Croes GC: The chemistry of isobutyraldehyde and its derivatives. Kingsport: Tennessee Eastman Co; 1953.

36. Atsumi S, Wu TY, Machado IM, Huang WC, Chen PY, Pellegrini M, Liao JC: Evolution, genomic analysis, and reconstruction of isobutanol tolerance in Escherichia coli. Mol Syst Biol 2010, 6:449.
37. Thomason LC, Costantino N, Court DL: E. coli genome manipulation by P1 transduction. Curr Protoc Mol Biol 2007, Chapter 1(Unit 1):17.

38. Li C, Wen A, Shen B, Lu J, Huang Y, Chang Y: FastCloning: a highly simplified, purification-free, sequence- and ligation-independent PCR cloning method. BMC Biotechnol 2011, 11:92.

doi:10.1186/1475-2859-11-90

Cite this article as: Rodriguez and Atsumi: Isobutyraldehyde production from Escherichia coli by removing aldehyde reductase activity. Microbial Cell Factories 2012 11:90.

\section{Submit your next manuscript to BioMed Central and take full advantage of:}

- Convenient online submission

- Thorough peer review

- No space constraints or color figure charges

- Immediate publication on acceptance

- Inclusion in PubMed, CAS, Scopus and Google Scholar

- Research which is freely available for redistribution 